\title{
Ring, Band or Suture in Tricuspid Annuloplasty for Functional Tricuspid Regurgitation; Which is Better and More Durable?
}

\author{
Ahmed Adas, ${ }^{1}$ Ahmed Elnaggar, ${ }^{2}$ Yehia Balbaa, ${ }^{2}$ Ahmed Elashkar, ${ }^{1}$ Hesham Alkady ${ }^{2}$ \\ ${ }^{1}$ Department of Cardiothoracic Surgery, Beni Suef University, Beni Suef, Egypt; ${ }^{2}$ Department of Cardiothoracic Surgery, Cairo \\ University, Egypt
}

\section{ABSTRACT}

Background: In this study, we evaluate different annuloplasty modalities to repair functional tricuspid regurgitation.

Patients and methods: Between January 2011 and January 2017, 200 patients with moderate or greater functional tricuspid regurgitation received tricuspid valve repair as part of primary surgeries on the left side of their cardiac valves. Of these, 39 patients received rings (Group A), 84 patients received bands (Group B), and 77 patients received suture annuloplasty (Group C).

Results: Two patients from Group C were operated on again, during the primary hospital stay due to severe symptomatic tricuspid regurgitation. The degrees of early postoperative tricuspid regurgitation - mean vena contracta and mean jet area - significantly were higher in Group C. During a mean follow-up period of $26 \pm 12.6$ months, 5 patients within Group C (6.85\%) and one patient in Group B $(1.3 \%)$ were operated on again with tricuspid valve replacement due to severe symptomatic tricuspid incompetence. Also during follow up, mean degrees of tricuspid regurgitation, mean vena contracta, and mean jet areas significantly were higher in Group C.

Conclusion: Patients who received rings followed by band annuloplasty had better early and late results with lower recurrence rates than those who received suture annuloplasty.

\section{INTRODUCTION}

The tricuspid valve always has received less attention from cardiac researches in comparison to left-sided cardiac valves with fewer studies describing the outcome of surgery [Parolari 2014]. The most common pathology affecting this valve is functional tricuspid regurgitation (FTR) as a consequence of annular dilatation, which occurs in close association with right ventricular dilatation and dysfunction as well as pulmonary hypertension seen with left-sided valve pathologies [Chan 2009]. Multiple annuloplasty modalities now are used to repair such pathology, including rigid or

Received April 17, 2019; received in revised form fuly 27, 2019; accepted fuly 29, 2019.

Correspondence: Hesham Alkady, Kasralaini str., Almanial, Cairo, Egypt; 00201006246611 (e-mail: beshamalqady@cu.edu.eg). flexible rings (complete or incomplete), sutures, and bands [Rogers 2009]. However, significant residual tricuspid insufficiency is associated with poor early- and late-postoperative outcomes and subsequent tricuspid valve surgery after previous cardiac surgery has a relatively high mortality [Nath 2004]. Also, recent reports greatly have debated the previous concepts that significant FTR would regress after treating left-sided valve lesions [Dreyfus 2005]. This is why there now is interest in widening the indications of FTR repair to include cases with moderate regurgitation and choosing the best and most durable method of repair at the time of the initial cardiac surgery. In this study, we evaluate our experience with different surgical techniques of FTR repair regarding early and late outcomes.

Patients and methods: Between January 2011 and January 2017, 200 patients with moderate or greater functional tricuspid regurgitation received tricuspid valve repair as part of primary surgeries on the left side of their cardiac valves at our university hospitals. Written informed consent was obtained from all patients for these planned operations. All patients received a 2D Doppler echocardiography before surgery with adequate assessment of the pathology of tricuspid valve (TV) and the severity of tricuspid regurgitation (TR). The severity of FTR was estimated in four grades using color Doppler and systolic flow in the inferior vena cava or hepatic veins as grade $1+$ (mild); grade $2+$ (moderate); grade 3+ (moderate to severe), and grade 4+ (severe). Moderate TR was defined when the width of vena contracta was $4-7 \mathrm{~mm}$ and the jet area was $5-10 \mathrm{~cm}^{2}$. Severe TR was considered when the width of vena contracta was $>7 \mathrm{~mm}$, and the jet area was $>10 \mathrm{~cm}^{2}$ with systolic flow reversal in hepatic veins. Also, TR was considered significant regardless of the degree of regurgitation when the tricuspid annulus diameter was $>40 \mathrm{~mm}$ [Matsunaga 2005]. Patients were categorized into three groups, according to the type of repair. Group A included 39 patients who underwent ring annuloplasty for repair of FTR, Group B consisted of 84 patients who received band annuloplasty, and Group C contained 77 patients who had suture annuloplasty.

Statistics: Data were abstracted from clinical records and coded to be entered into the statistical package SPSS (Statistical Package for the Social Sciences). Quantitative data were summarized using mean \pm standard deviation, while categorical data were presented as frequency (count) and relative frequency (percentage). The significance of the association between categorical data was conducted using the 
Table 1. Preoperative patient characteristics

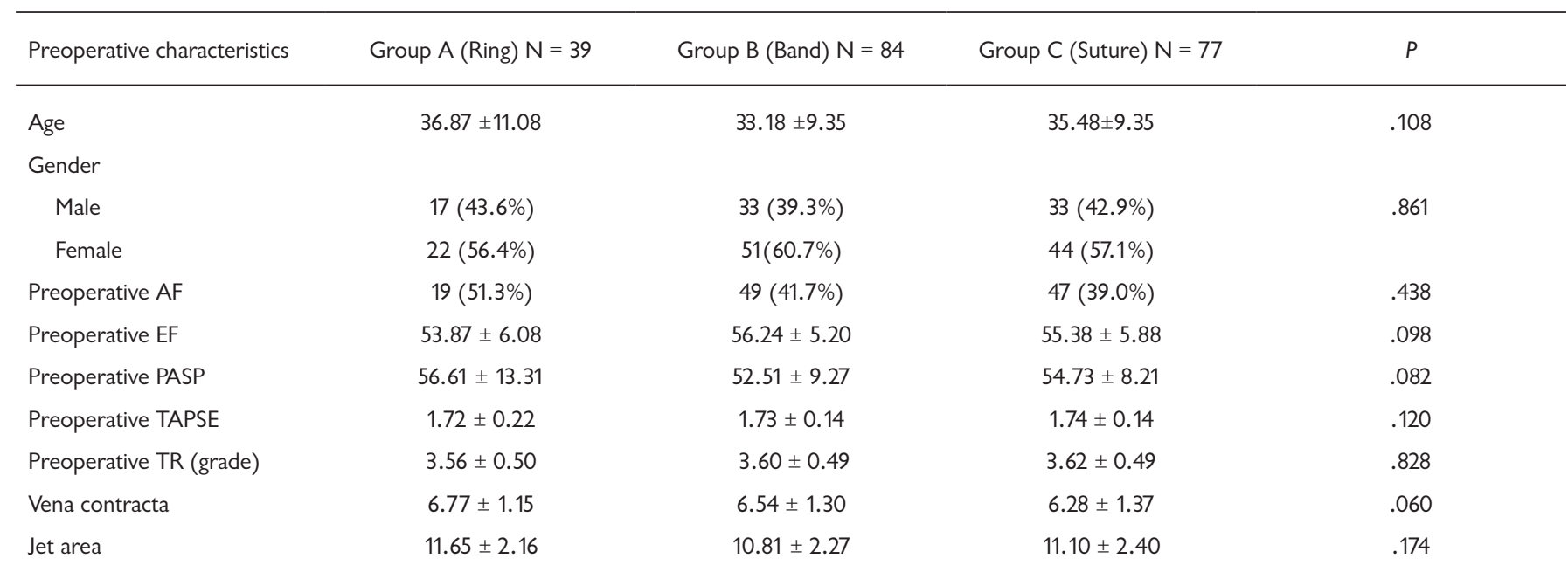

Table 2. Operative data

\begin{tabular}{lccc}
\hline Preoperative characteristics & Group A (Ring) N=39 & Group B (Band) N=84 & Group C (Suture) N=77 \\
\hline Mode of repair & & & \\
Beating heart & $4(10.3 \%)$ & $6(7.1 \%)$ & $36(46.8 \%)$ \\
Arrested heart & $35(89.7 \%)$ & $78(92.9 \%)$ & $41(53.2 \%)$ \\
Bypass time (min) & $133.44 \pm 33.33$ & $122.77 \pm 36.33$ & $114.94 \pm 30.20$ \\
Cross-clamp time (min) & $102.2 \pm 26.24$ & $100.00 \pm 33.25$ & $89.87 \pm 27.53$
\end{tabular}

Table 3. Postoperative data

\begin{tabular}{lcccc}
\hline Preoperative characteristics & Group A (Ring) N=39 & Group B (Band) N=84 & Group C (Suture) N=77 \\
\hline Mechanical ventilation time & $11.08 \pm 8.57$ & $11.60 \pm 7.49$ & $12.00 \pm 8.01$ & .196 \\
ICU stay & $2.48 \pm 1.44$ & $2.24 \pm 0.57$ & $5.38 \pm 0.81$ & .080 \\
Postoperative EF & $51.24 \pm 5.09$ & $53.55 \pm 4.29$ & $32.01 \pm 5.95$ & .430 \\
Postoperative PASP & $35.55 \pm 9.27$ & $33.83 \pm 5.60$ & $1.80 \pm 0.22$ & .09 \\
Postoperative TAPSE & $1.77 \pm 0.25$ & $1.74 \pm 0.170$ & $1.17 \pm 0.90$ & .221 \\
Postoperative TR (grade) & $0.76 \pm 0.58$ & $0.84 \pm 0.78$ & $2.47 \pm 1.72$ & .001 \\
Postoperative VC & $1.22 \pm 1.03$ & $1.59 \pm 1.56$ & $3.86 \pm 2.72$ & .001
\end{tabular}

student's t-test while the chi-square test was used for comparison of categorical parameters. One-way ANOVA test was used to analyze the data between the study groups and a $P$-value less than 0.05 was considered statistically significant. Kaplan-Meier curves were used to determine the overall survival rate and freedom from significant tricuspid regurgitation.

Surgical techniques: All patients were operated on through full median sternotomy. Cardiopulmonary bypass (CPB) was established after cannulating the ascending aorta and both cavae. Myocardial protection was performed using antegrade with or without retrograde intermittent cold blood cardioplegia together with systemic cooling to $32^{\circ} \mathrm{C}$. Left-sided valvular lesions were managed after aortic cross-clamping. De-airing of the left side of the heart was then done through filling the left atrium and ventricle with blood aided by the Valsalva maneuver and suction of the aortic root. Cavae were snared, and the right atrium was obliquely opened $1 \mathrm{~cm}$ parallel to the atrioventricular groove to expose the TV. The choice of the type of tricuspid repair as well as whether to do it on an arrested or beating heart while still on pump was carried out, 


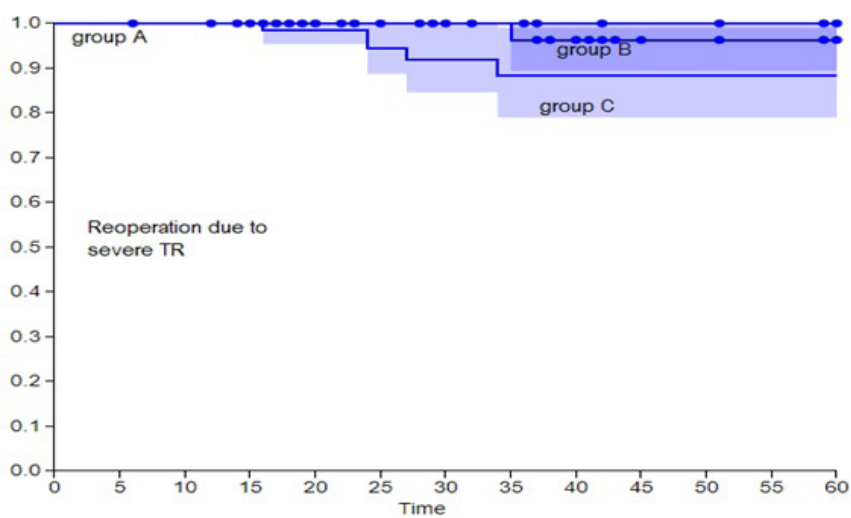

Kaplan-Meier curve for rate of reoperation among different groups

according to the surgeon's preference and self-confidence with the technique.

Ring annuloplasty was performed using CarpentierEdwards incomplete rigid rings (Baxter Healthcare Corp., Edward Division, Santa Ana, CA) whose size was determined, according to the base of the septal leaflet. Tricuspid rings were fixed to the annulus with interrupted non-pledgeted $2 / 0$ non-absorbable (Ethibond) sutures avoiding the area of the AV node. Band annuloplasty was done using a strip of Dacron tube graft with a fixed length of $5 \mathrm{~cm}$ and a width of $3 \mathrm{~mm}$. The band was attached to the annulus by interrupted nonpledgeted 2/0 Ethibond sutures, starting from the anteroseptal commissure to end at the posteroseptal commissure. Suture annuloplasty was carried out by the De Vega technique using a pledgeted 2/0 Ethibond suture, starting from the anteroseptal commissure and ending at the posteroseptal commissure. Both lines then were tightened over a number 27 sizer and tied over a pledget.

\section{RESULTS}

Preoperative data: In our study, the mean age was $34.7 \pm$ 9.7 years. Eight-three (41.5\%) patients were male, and 117 (58.5\%) patients were female. Eight-five (42.5\%) patients had atrial fibrillation (AF) preoperatively while the remaining 115 (57.5\%) had sinus rhythm. A high number - 192 (96\%) patients - complained of dyspnea functional class III, according to New York Heart Association (NYHA) classification, and $8(4 \%)$ were in dyspnea functional class IV. Other signs of right-sided heart failure occurred in the form of lower limb edema and ascites in $2(1 \%)$ patients. The mean LV ejection fraction $(\mathrm{EF})$ was $55.4 \pm 5.6 \%$, while the mean pulmonary artery systolic pressure (PASP) was $54.1 \pm 9.89 \mathrm{mmHg}$. The mean tricuspid annular plane systolic excursion (TAPSE) was $1.72 \pm .16$. The mean preoperative degree of TR was $3.6 \pm$ 0.49 with a mean vena contracta of $6.25 \pm 1.3$ and a mean jet area of $11.08 \pm 2.31$. The 3 groups were matched regarding the preoperative patient characteristics (Table 1).

Operative data: Ninety-one $(45.5 \%)$ patients received in addition to tricuspid valve repair mitral valve replacement; mitral valve repair was done in 34 (17\%) patients, 45 (22.5\%) patients received aortic and mitral valve replacement, and 30 $(15 \%)$ patients underwent aortic valve replacement and mitral valve repair. The mean total bypass time was $121.84 \pm 34$ minutes, while the mean cross-clamp time was $96.54 \pm 30.1$ minutes. Forty-six (23\%) patients had TV repair using beating heart technique, while the remaining 154 (77\%) patients underwent TV repair by arrested heart technique. As the choice of repair technique whether ring, suture or band was according to surgical preference, significantly more patients in the suture group (Group C) could be done on beating heart, due to the simplicity of the technique. This was reflected in both the cross-clamp and bypass times that significantly were longer in the ring (Group A) and band techniques (Group $\mathrm{B})$, due to the additional time needed for placing the annulus sutures and tying them (Table 2).

Postoperative data: The mean mechanical ventilation time was $12.4 \pm 7.9$ hours, while the mean ICU stay was $2.39 \pm$ 0.91 days. Five patients needed a pacemaker in the early postoperative period, but none required a permanent pacemaker before discharge. The remaining patients showed no change in the preoperative heart rhythm. Two patients developed cerebrovascular strokes in the form of hemiplegia and were discharged to a rehabilitation facility. Three patients were operated on again in the primary hospitalization, one due to severe mitral regurgitation following mitral repair and 2 patients with severe TR and signs of right-sided heart failure (ascites and lower limb edema) following suture annuloplasty. Only 3 patients $(1.5 \%)$ died in the early postoperative period. The cause of death for these patients was low cardiac output syndrome not related to the tricuspid valve repair, which was refractory to maximum inotropic support and intra-aortic balloon pump (IABP) application. Echocardiography was done to all patients before discharge and showed a mean early postoperative EF of $52.5 \pm 5.1 \%$, mean PASP of $34.58 \pm 6.64$ $\mathrm{mmHg}$, mean TAPSE of $1.84 \pm .17$, and mean degree of TR of $0.892 \pm 0.875$, with a mean vena contracta of $1.81 \pm 1.66$ and mean jet area of $3.04 \pm 2.7$. The degree of TR, mean vena contracta and mean jet area significantly was highest in Group C. These values were higher in Group B than Group A, but not statistically significant (Table 3 ).

Follow up: One hundred ninety-five $(97.5 \%)$ patients survived until discharge. Of that figure, 180 (92\%) patients could be followed up with echocardiography in 6- to 8-month intervals for a period ranging between 8 months and 60 months (mean $26 \pm 12.6$ months). These patients were distributed as 32 cases from Group A, 75 from Group B, and 73 from Group C. The remaining 15 patients could not be followed up on due to lack of compliance. Five patients within Group $\mathrm{C}$ were operated on again with tricuspid valve replacement, due to severe symptomatic (ascites and lower limb edema) TR during the follow-up period (6.85\%), while in Group B only one $(1.3 \%)$ patient received a new tricuspid valve due to severe symptomatic TR. In Group A, no surgeries were reperformed, due to severe TR. This difference statistically was significant among the 3 groups $(P=.004)$ (Figure 1$)$.

Mean degrees of TR during the echocardiographic follow up in Groups A, B, and C were $0.70 \pm 0.5,0.91 \pm 0.80$, and 
$1.09 \pm 1.05$, respectively with statistically significant differences among the 3 groups $(P=.008)$.

Also, the means of vena contracta were $1.24 \pm 0.90,1.57 \pm$ 1.47 , and $1.90 \pm 1.60$ in Groups A, B, and C, respectively with a significant $P$-value among the 3 groups of .006. Similarly, mean jet areas were $2.64 \pm 2.42,2.94 \pm 2.77$, and $3.5270 \pm$ 3.07 in Groups A, B, and C, respectively with a significant $P$-value of .004. No patient died from a cardiac cause, during the follow-up period.

\section{DISCUSSION}

Many surgeons now encourage the repair of moderate or greater FTR with surgery on left-sided cardiac valves especially in the presence of annular dilatation $>40 \mathrm{~mm}$, severe pulmonary hypertension, $\mathrm{AF}$, and signs of right ventricular dysfunction [Matsunaga 2005; Navia 2012; Van de Veire 2011; Bianchi 2009]. In our study we have chosen to repair FTR if moderate or greater according to width of vena contracta, jet area and systolic flow reversal in hepatic veins or if annular diameter exceeded $40 \mathrm{~mm}$ regardless of the degree of TR.

Several techniques have been used to treat FTR. Suturebased annuloplasty like De Vega and bicuspidalization appeared first and are still used. These techniques are easy, fast, cost-effective, and preserve the anatomy as well as the flexibility of the annulus [Bernal 2004]. The De Vega technique is more commonly employed and appears to be superior to bicuspidalization [Naqshband 2010]. On the other, hand rings appeared later and prove to give good long-term results due to the stabilization of the annulus. Both flexible and rigid rings are approved with contradictory opinions regarding the superiority of any type over the other [Zhu 2013]. In general, rigid rings have been more reported to achieve better freedom from recurrent TR than flexible rings [Navia 2010]. Although, it has been reported that flexible rings, as mentioned by some studies, preserve the physiologic annulus contraction [Gatti 2001]. Many studies documented better longterm results using ring annuloplasty with better freedom from recurrent TR and right ventricular failure as well as survival [Tang 2006; McCarthy 2004; Guenther 2013]. Nevertheless, other studies showed better results or at least no difference when using suture annuloplasty [Morishita 2002].

Ring annuloplasty seems to provide longer annular stabilization over time while the De Vega technique may widen the anteroseptal commissure by pulling it away from the anterior annulus and distort the anterior annulus throwing it into folds resulting in an uneven coaptation with the septal leaflet. Finally, the De Vega suture may later avulse or break [Wang 2008]. Band annuloplasty similarly is reported in some studies to give results close to the ring and more superior to the De Vega [Calafiore 2011].

Unfortunately, these studies comparing different repair techniques enclosed heterogeneous groups concerning age, gender, concomitant procedures, and echocardiographic data. In our study, the 3 groups were matched, regarding the preoperative patient characteristics which made the postoperative comparison more reliable. According to our study, ring annuloplasty significantly was associated with better early and late results as well as more freedom from late recurrent significant TR. Also, according to our results, band annuloplasty gives a better outcome than De Vega, although non-significant, and therefore is recommended when rings are not available. On the other hand, ring or band placement requires more time and many surgeons worldwide, as well as ours, prefer to apply them on an arrested heart, prolonging both bypass and crossclamp times.

\section{CONCLUSION}

Our study showed that patients who received ring annuloplasty followed by band annuloplasty for the treatment of FTR concomitant to left-sided valve lesions had better early and late results with lower recurrence rates than those who received suture annuloplasty.

\section{REFERENCES}

Bernal JM, Gutierrez-Morlote J, Llorca J, et al. 2004. Tricuspid valve repair: an old disease, a modern experience. Ann Thorac Surg 78: 2069-74.

Bianchi G, Solinas M, Bevilacqua S, Glauber M. 2009. Which patient undergoing mitral valve surgery should also have the tricuspid repair? Interact Cardiovasc Thorac Surg 9:1009-20.

Calafiore AM, Di Mauro M. 2011. Tricuspid Valve Repair-Indications and Techniques: Suture Annuloplasty and Band Annuloplasty. Operative Techniques in Thoracic and Cardiovascular Surgery 16:86-96.

Chan KM, Zakkar M, Amirak E, Punjabi PP. 2009. Tricuspid valve disease: pathophysiology and optimal management. Prog Cardiovasc Dis 51:482-6.

Dreyfus GD, Corbi PJ, Chan KM, Bahrami T. 2005. Secondary tricuspid regurgitation or dilatation: which should be the criteria for surgical repair? Ann Thorac Surg 79:127-32.

Gatti G, Maffei G, Lusa AM, Pugliese P. 2001. Tricuspid Valve Repair With the Cosgrove-Edwards Annuloplasty System: Early Clinical and Echocardiographic Results. Ann Thorac Surg 72:764-7.

Guenther T, Mazzitelli D, Noebauer C, et al. 2013. Tricuspid valve repair: is ring annuloplasty superior? Eur J Cardiothorac Surg 43:58-65.

Matsunaga A, Duran CM. 2005. Progression of tricuspid regurgitation after repaired functional ischemic mitral regurgitation. Circulation 112:I453-7.

McCarthy PM, Bhudia SK, Rajeswaran J, et al. 2004. Tricuspid valve repair: Durability and risk factors for failure. J Thorac Cardiovasc Surg $127: 674-85$

Morishita A, Kitamura M, Noji S, et al. 2002. Long-term results after De Vega's tricuspid annuloplasty. J Cardiovasc Surg (Torino) 43:773-7.

Naqshband MS, Abid AR, Akhtar RP, Waheed A, Khan JS. 2010. Functional tricuspid regurgitation in rheumatic heart disease: surgical options. Ann Thorac Cardiovasc Surg 16: 417-25.

Nath J, Foster E, Heidenreich PA. 2004. Impact of tricuspid regurgitation on long-term survival. J Am Coll Cardiol 43:405-9. 
Navia JL, Brozzi NA, Klein AL, et al. 2012. Moderate tricuspid regurgitation with left-sided degenerative heart valve disease: to repair or not to repair? Ann Thorac Surg 93:59-69.

Navia JL, Nowicki ER, Blackstone EH, et al. 2010. Surgical management of secondary tricuspid valve regurgitation: annulus, commissure, or leaflet procedure? J Thorac Cardiovasc Surg 139:1473-82.

Parolari A, Barili F, Pilozzi A, Pacini D. 2014. Ring or Suture Annuloplasty for Tricuspid Regurgitation? A Meta-Analysis Review. Ann Thorac Surg 98:2255-63.

Rogers JH, Bolling SF. 2009. The tricuspid valve: Current perspective and evolving management of tricuspid regurgitation. Circ. 119:2718-25.15.

Tang GH, David TE, Singh SK, et al. 2006. Tricuspid valve repair with an annuloplasty ring results in improved long-term outcomes. Circulation 114:1577-81.

Van de Veire NR, Braun J, Delgado V, et al. 2011. Tricuspid annuloplasty prevents right ventricular dilatation and progression of tricuspid regurgitation in patients with tricuspid annular dilatation undergoing mitral valve repair. J Thorac Cardiovasc Surg 141:1431-9.

Wang G, Sun Z, Xia J, et al. 2008. Predictors of secondary tricuspid regurgitation after left-sided valve replacement. Surg Today 38:778-83.

Zhu TY, Wang JG, Meng X. 2013. Is a rigid tricuspid annuloplasty ring superior to a flexible band when correcting secondary tricuspid regurgitation? Interact Cardiovasc Thorac Surg 17:1009-14. 